\title{
Evidence for Elton's diversity-invasibility hypothesis from belowground
}

\author{
Zhije Zhang (D), ${ }_{1}^{1}$ Yanje Liu (DD , ${ }^{2,5}$ Caroline Brunel (D), 3,4 and Mark van Kleunen (iD) 1,3 \\ ${ }^{1}$ Ecology, Department of Biology, University of Konstanz, Konstanz 78464 Germany \\ ${ }^{2}$ Key Laboratory of Wetland Ecology and Environment, Northeast Institute of Geography and Agroecology, Chinese Academy \\ Sciences, Changchun 130102 China \\ ${ }^{3}$ Zhejiang Provincial Key Laboratory of Plant Evolutionary Ecology and Conservation, Taizhou University, Taizhou 318000 China \\ ${ }^{4}$ IRD, IPME, 911 Avenue Agropolis, BP 64501, Montpellier 34394 France
}

Citation: Zhang, Z., Y. Liu, C. Brunel, and M. van Kleunen. 2020. Evidence for Elton's diversity-invasibility hypothesis from belowground. Ecology 101(12):e03187. 10.1002/ecy.3187

\begin{abstract}
Sixty year ago, Charles Elton posed that species-rich communities should be more resistant to biological invasion. Still, little is known about which processes could drive the diversity-invasibility relationship. Here we examined whether soil-microbe-mediated apparent competition on alien invaders is more negative when the soil originates from multiple native species. We trained soils with five individually grown native species and used amplicon sequencing to analyze the resulting bacterial and fungal soil communities. We mixed the soils to create trained soils from one, two or four native species. We then grew four alien species separately on these differently trained soils. In the soil-conditioning phase, the five native species built species-specific bacterial and fungal communities in their rhizospheres. In the test phase, it did not matter for biomass of alien plants whether the soil had been trained by one or two native species. However, the alien species achieved 11.7\% (95\% CI: 3.7-20.1\%) less aboveground biomass when grown on soils trained by four native species than on soils trained by two native species. Our results revealed soil-microbes-mediated apparent competition as a mechanism underlying the negative relationship between diversity and invasibility.
\end{abstract}

Key words: apparent competition; diversity; invasibility; mutualists; pathogens; plant invasion; plantsoil feedback; soil legacy; soil microbes; soil mixture.

\section{INTRODUCTION}

In the last centuries, most regions of the world have been invaded by alien organisms (Dawson et al. 2017, Pyšek et al. 2017, Seebens et al. 2018). The increasing numbers of naturalized alien species have stimulated discussion on how to increase resistance of ecological communities to biological invasions. Elton (1958) posed that species-rich communities are more resistant to invasion. Support for Elton's diversity-invasibility hypothesis comes from experiments, particularly on plants (Levine 2000, Levine et al. 2004). Theoretical models usually ascribed this relationship to a lack of available resources in species-rich communities (Case 1990, Byers and Noonburg 2003), likely because Elton (1958) introduced his hypothesis with several examples in which resource competition was likely to determine invasibility (i.e., the probability of an alien species to establish and persist in a native community). However, most experimental studies focused on the relationship between diversity and invasibility, but not on the potential underlying

Manuscript received 23 November 2019; revised 26 June 2020; accepted 14 July 2020; final version received 14 August 2020. Corresponding Editor: Hannah L. Buckley.

${ }^{5}$ Corresponding Author. E-mail: liuyanjie@iga.ac.cn mechanisms. Moreover, Elton noted that "there may be many indirect influences, acting through other species like parasites or enemies" (Elton 1958, P122). Yet, it remains unknown whether such apparent competition (Holt 1977), mediated by higher trophic levels, could drive the relationship between diversity and invasibility.

The last two decades have seen an increase in evidence for the importance of soil microbes in mediating plants coexistence. Numerous studies found that plants can modify the soil microbial communities by releasing organic matter and other chemical compounds, which influence performance of the plants that later grow on the same soil (Bever et al. 1997, Kulmatiski et al. 2008, Bennett and Klironomos 2019). However, few studies have tested how species-rich communities affect later plants through modifying soil microbial communities (but see Müller et al. 2016, Heinen et al. 2018). This is surprising, given that natural plant communities typically contain multiple intermingled species that each might have different effects on microbes. As soil microbes include different functional groups that can increase (mutualists) or decrease (pathogens) plant growth (Morris et al. 2007), plant-diversity-mediated changes in the microbial community might affect establishment of introduced plant species. Until now, we still know little about whether species-rich plant 
communities better resist alien plants through soil microbes than species-poor communities do.

Several hypotheses offer insights into how soil-microbes-mediated apparent competition could affect the diversity-invasibility relationship, but they predict different patterns. First, the amplification-effect hypothesis proposes that species-rich communities of plant species harbor a greater diversity and abundance of pathogens (Hudson et al. 2006, Keesing et al. 2006). This increases the likelihood that some of those pathogens will affect later plants more negatively. Following this logic, speciesrich communities should be better able to resist alien plants. Second, the dilution-effect hypothesis proposes that species-rich communities reduce the abundance of highly susceptible hosts (Schmidt and Ostfeld 2001, Ostfeld and Keesing 2012). This reduces the prevalence of pathogens, and will affect later plants less negatively. Following this logic, species-rich communities should be less able to resist alien plants. Third, the enemy-release hypothesis proposes that alien plants are released from enemies, such as pathogens (Mitchell and Power 2003). Following this logic, even when the diversity of plant communities affects pathogens, it might not strongly affect resistance of the plant communities to alien plants.

While the hypotheses listed above focus on pathogens, soil microbes also include mutualists. Consideration of mutualists would result in similar but opposite predictions. First, if plant diversity amplifies mutualists, species-rich communities will be more likely to include mutualists that facilitate alien plants, and these communities will thus be less able to resist aliens (Reinhart and Callaway 2006). Second, if plant diversity dilutes the prevalence of mutualists, species-rich communities will be better able to resist alien plants. Third, if alien plants miss their mutualists (Mitchell et al. 2006) or are not tightly associated with mutualists (Pyšek et al. 2019), plant diversity will not affect resistance to alien plants. Given the contrasting predictions, empirical tests are necessary to test whether and how soil microbes contribute to the diversity-invasibility relationship.

Here, we conducted an experiment with five native plant species and four alien plant species to test whether the diversity-invasibility relationship may be mediated by soil microbes. We first grew each of the five native species individually to train soils. Then, we mixed soil samples from one, two, or four native species, and grew one of the four alien species on the soil mixture (Fig. 1a). As the approach of mixing soil samples per se might affect plants by increasing diversity of soil microbes, even if the soil samples come from the same species (Reinhart and Rinella 2016, Rinella and Reinhart 2018, Gundale et al. 2019, Peacher and Meiners 2020), we additionally created soil mixtures for single native species from one, two or four different individuals, and then grew one of the four alien species on the soil mixture (Fig. 1b). We tested the following specific questions: (1) Does diversity of native plant species affect growth of alien plant species through soil- microbes-mediated competition? And (2) does the number of samples used to create the soil mixture affect growth of alien plant species (i.e., does the approach of mixing soil samples per se affect plants)?

\section{Materials And Methods}

\section{Study species}

We conducted a greenhouse experiment in which we used five herbaceous species that are native to Germany (Dactylis glomerata, Leontodon autumnalis, Lotus corniculatus, Plantago media, Salvia pratensis) to condition the soil, and four alien herbs (Epilobium ciliatum, Lolium multiflorum, Senecio inaequidens, Vicia villosa) as test species. We used multiple aliens as test species to increase our ability to generalize the results (van Kleunen et al. 2014). The classification of the status (native or alien) of the nine species was based on the FloraWeb database (Bundesamt für Naturschutz 2003). All species are widespread in Germany and can be locally abundant. So, the four alien species can be classified as naturalized (and probably invasive; sensu Richardson et al. 2000b) in Germany. The nine species mainly occur in grasslands and overlap in their distributions (Bundesamt für Naturschutz 2003), so that they are likely to co-occur in nature. Seeds of the native species were obtained from Rieger-Hofmann $\mathrm{GmbH}$ (Blaufelden-Raboldshausen, Germany), and seeds of alien species were obtained from the Botanical Garden of the University of Konstanz.

\section{Experimental setup}

The experiment consisted of three steps (Fig. 1a). In step 1 , we had the soil-conditioning phase, in which we trained the soils by growing each of the five native species individually on the soils. In step 2, we collected soil samples from the soil-conditioning phase, and created different soil-mixture treatments: soil mixtures trained by one, two, or four species. In step 3, the test phase, we grew the four alien species individually on each soil mixture and determined biomass production. Details on each of these steps are given below.

Soil-conditioning phase (step 1).-On 18 or 27 June 2018, we sowed seeds of the five native species. Each species was sown into a separate tray $(10 \times 10 \times 5 \mathrm{~cm})$ filled with potting soil (Topferde; Einheitserde Co., Sinntal-Altengronau, Germany). The soil and seeds were not sterilized. Because we wanted the different species to be in similar developmental stages at the beginning of the experiment, we sowed the species at different times (Appendix S1: Table S1), according to their germination speed known from previous experiments. We then placed the trays with seeds in a greenhouse under natural light conditions, with a temperature between $18^{\circ} \mathrm{C}$ and $25^{\circ} \mathrm{C}$.

On 9 July 2018, we transplanted 140 seedlings of each native species into 1.5 - $\mathrm{L}$ pots filled with $25 \%$ field soil, 

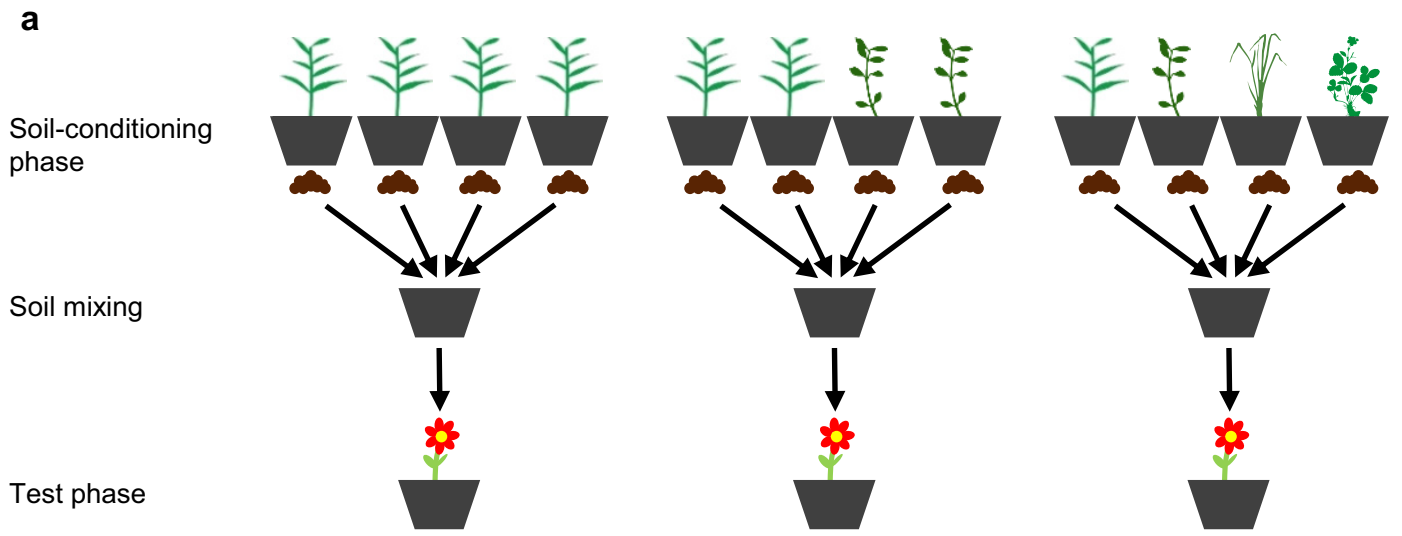

\section{b \\ Soil-conditioning phase}

Soil mixing

Test phase
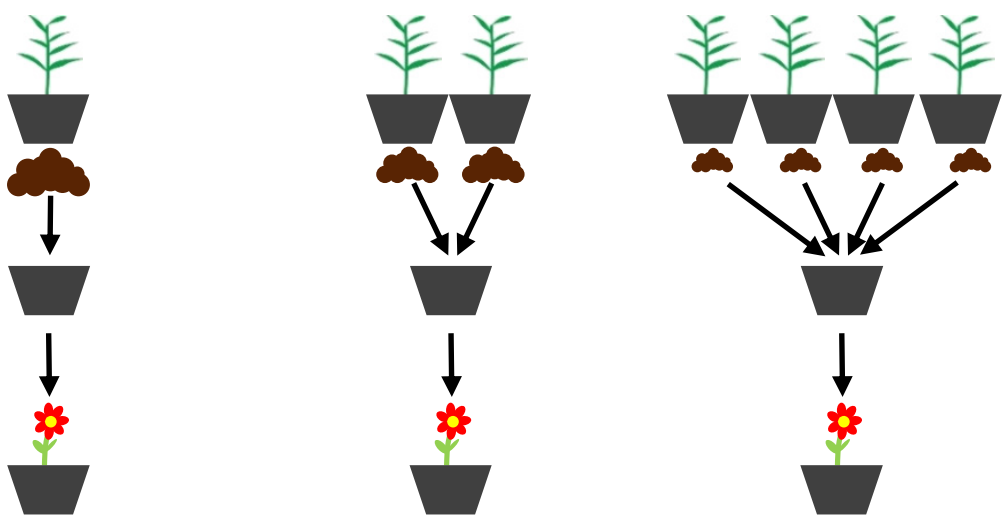

FIG. 1. Graphical illustration of the experimental design. (a) In the experiment for testing the effect of native plant diversity on alien plants, soil samples trained by one, two, or four native species were collected from four individuals (soil-conditioning phase). (b) In the experiment for testing the effect of mixing soil, soil samples trained by one of the five native species were collected from one, two, or four individuals (soil-conditioning phase). Note that the treatment with four individuals in the experiment on the effect of mixing soil (the right side in b) was also part of the experiment on the effect of native plant diversity (the left side in a). Then, the soil samples were used as inoculum, and mixed with sand and vermiculite (soil mixing). In each pot, one plant of each alien species was grown (test phase). The total amount of soil used to inoculate each pot was constant (i.e., $160 \mathrm{~mL}$ ). Five native species and four alien species were used in the soil-conditioning phase and test phase, respectively. [Color figure can be viewed at wileyonlinelibrary.com]

$37.5 \%$ nonsterilized sand, and $37.5 \%$ nonsterilized vermiculate. Each pot contained one single seedling. The field soil, which served as inoculum to provide a live soil biotic community, had been collected from a grassland site in the Botanical Garden of the University of Kon$\operatorname{stanz}\left(47.69^{\circ} \mathrm{N}, 9.18^{\circ} \mathrm{E}\right)$. The soil had been sieved through a $1-\mathrm{cm}$ mesh to remove plant material and large stones. We placed each of the 700 pots on its own plastic dish to preserve water and to avoid cross-contamination through soil solutions leaking from the pots. We replaced seedlings that died within two weeks after transplanting by new ones. The pots were randomly allocated to positions in four greenhouse compartments $\left(23^{\circ} \mathrm{C}\right.$ : $18^{\circ} \mathrm{C}$ day: night temperature, no additional light), fertilized with an NPK water soluble fertilizer (Universol Blue; Everris, Nordhorn, Germany) at a concentration of $1 \% \mathrm{~m} / \mathrm{v}$ seven times $(100 \mathrm{~mL}$ fertilizer per pot per time), watered as needed, and re-randomized twice.
From 22 to 26 October 2018, we collected soils from each pot by first cutting the shoot and then removing the roots from the soil by sieving it through a $5-\mathrm{mm}$ mesh. We randomly selected 120 pots of soil for each of the five species (600 pots in total), and used them to create the different soil-mixture treatments.

Soil-mixture treatments (step 2).-To create a speciesdiversity gradient, we mixed soil samples from the soilconditioning phase to create three treatment levels, which we call diversity-1, diversity-2, and diversity-4 (Fig. 1a). In the diversity-1 treatment, we collected a total of $160 \mathrm{~mL}$ soil from four different pots of the same species (i.e., $40 \mathrm{~mL}$ from each pot). In the diversity- 2 treatment, we collected a total of $160 \mathrm{~mL}$ soil from four different pots of two species (i.e., two pots per species). In the diversity-4 treatment, we collected a total of $160 \mathrm{~mL}$ soil from four different pots of four species (i.e., 
one pot per species). Then, the $160 \mathrm{~mL}$ of soil was mixed with $220 \mathrm{~mL}$ nonsterilized sand and $220 \mathrm{~mL}$ nonsterilized vermiculite, and put into 0.6-L pots, which were then used in the test phase described below. As we had a total of five native species in the soil-conditioning phase, this resulted in five possible species or combinations for diversity- 1 and for diversity 4 , but 10 possible combinations for diversity-2. To have equal numbers of combinations for each diversity treatment, we therefore used only 5 out of the 10 possible combinations for diversity-2. We did this in such a way that each species was included in two of the five combinations. Consequently, we had five species or species combinations for each diversity treatment. Each of those combinations was replicated eight times, such that we had a total of 120 pots for the test phase (see Appendix S1: Table S2 for combinations of native species that were used). Once we had collected soil from a pot of the soil conditioning phase, we did not use that pot again. This was done to avoid pseudoreplication (Hurlbert 1984), because this way no two pots in the test phase shared soil from the same pot of the soil-conditioning phase (i.e., they were independent).

In the diversity-1 treatment, we used soil of one single plant species, and in the diversity- 2 treatment, we used soil of two plant species. Nevertheless, we still collected the soil from four different pots, just like in the diversity4 treatment. We did this because recent studies argued that the approach of mixing soil samples per se can affect plants by increasing diversity of soil biota (Reinhart and Rinella 2016, Rinella and Reinhart 2018). More specifically, the composition of the soil biotic community can vary substantially across centimeters given their immense diversity (Decaëns 2010). So, microbes from different soil samples could differ in their identity, even when trained by the same plant species. This means that mixing soil samples might increase the diversity of mutualists and/or pathogens. Therefore, by consistently mixing soil samples from four pots for all diversity treatments, we reduced the potential side effects resulting from soil mixing.

Furthermore, to explicitly test whether the mixing of soil samples from different individuals of the same species affects plants in test phase, we added two further one-species (i.e., diversity-1) treatments (Fig. 1b). In one of the treatments, we collected a total of $160 \mathrm{~mL}$ soil from just one pot, and in the other treatment, we collected a total of $160 \mathrm{~mL}$ soil from two different pots $(80 \mathrm{~mL}$ from each pot). Each of these two additional treatments was replicated eight times for each of the five native species, such that we had a total of 80 additional pots for the test phase.

Test phase (step 3).-Between 9 and 18 October 2018 (Appendix S1: Table S1), we sowed the four alien species into trays filled with the same type of potting soil as in the soil conditioning phase. The soil and seeds were not sterilized. On 27 October 2018, we transplanted the seedlings into the $0.6-\mathrm{L}$ pots prepared in the soil mixture phase. We assigned the seedlings in such a way that each alien species was replicated twice on each soil of a native species or native species combination (totaling 50 pots per test species). We placed each of the 200 pots on its own plastic dish. The pots were randomly allocated to position in a greenhouse compartment $\left(20^{\circ} \mathrm{C}: 15^{\circ} \mathrm{C}\right.$ day: night temperature, $14 \mathrm{~h}: 10 \mathrm{~h}$ day:night light), and rerandomized once during the experiment. The plants were fertilized with a water soluble NPK fertilizer (Universol Blue, Everris) at a concentration of $1 \% \mathrm{~m} / \mathrm{v}$ four times (100 $\mathrm{mL}$ fertilizer per pot per time). The fertilizer addition might have affected the microbial communities (in 't Zandt et al. 2019), but as all pots received the same amount of fertilizer, it should not affect comparability of the different treatments. By homogenizing the soils in the soil-mixture treatments, inoculating a small amount $(26.7 \% \mathrm{v} / \mathrm{v})$ of trained soil and fertilizing the plants throughout the experiment, we likely removed differences in abiotic properties between treatments. Therefore, any differences, if found, would be mainly due to differences in biotic properties of the soils. On 18 December 2018, seven weeks after the start of the test phase, we harvested all aboveground plant parts, and then washed the roots free from soil. The biomass was dried at $70^{\circ} \mathrm{C}$ for one week to constant mass, and weighed to the nearest milligram.

Soil sampling, DNA extraction, amplicon sequencing, and bioinformatics. - From 22 to 26 October 2018, when we harvested the trained soil, we randomly selected six pots for each of the five native species. For each of these pots, 10-20 mL rhizosphere-soil was collected into a sterile $50-\mathrm{mL}$ cylindrical tube, and stored at $-80^{\circ} \mathrm{C}$ until required for DNA extraction. We extracted DNA from $0.25 \mathrm{~g}$ of each soil sample using the DNeasy PowerSoil Kit (Qiagen, Hilden, Germany), following the manufacturer's protocol.

PCR amplifications and amplicon sequencing were then performed by Novogene (Beijing, China). In brief, V3-V4 region of bacterial 16S rDNA gene was amplified in triplicate with the universal primers 341F/806R (forward primer: 5'-CCTAYGGGRBGCASCAG-3'; reverse primer: 5'-GGACTACNNGGGTATCTAAT-3') (Klindworth et al. 2013). The ITS2 region of fungal rDNA gene was amplified in triplicate with the specific primers ITS3 (5'-GCATCGATGAAGAACGCAGC- $\left.3^{\prime}\right)$ and ITS4 (5'-TCCTCCGCTTATTGATATGC-3') (Orgiazzi et al. 2012). The amplification was conducted in $25-\mu \mathrm{L}$ reactions under the following conditions: initial denaturation at $95^{\circ} \mathrm{C}$ for 3 minutes, followed by 30 cycles at $95^{\circ} \mathrm{C}$ for $30 \mathrm{~s}, 55^{\circ} \mathrm{C}$ for $30 \mathrm{~s}, 72^{\circ} \mathrm{C}$ for $45 \mathrm{~s}$, and a final extension step of 5 minutes at $72^{\circ} \mathrm{C}$. All PCR reactions were carried out with Phusion High-Fidelity PCR Master Mix (New England Biolabs, Ipswich, Massachusetts, USA). PCR products were mixed in equidensity ratios. Then, mixtures of PCR products were purified with Gel Extraction Kit (Qiagen). The libraries were generated with NEBNext Ultra DNA Library Prep Kit for 
Illumina and analyzed using the NovaSeq Illumina platform (Illumina, San Diego, CA, USA).

We processed the raw sequences with the DADA2 pipeline (Callahan et al. 2016), which is designed to resolve exact biological sequences (Amplicon Sequence Variants [ASVs]) from Illumina sequence data and does not involve sequence clustering (Callahan et al. 2017). The detailed process was described in Brunel et al. (2019). In short, we removed primers and adapter with the cutadapt package (Martin 2011), merged paired-end sequences, and removed chimeras. Then, we assigned the sequences to taxonomic groups using the SILVA (Quast et al. 2013) and the UNITE (Nilsson et al. 2018) taxonomic databases for bacteria and fungi, respectively. Last, we rarefied bacteria and fungi to 30,000 and 10,000 reads, respectively, to account for differences in sequencing depths. One sample that did not have enough reads for bacteria and two samples that did not have enough PCR product for fungi were excluded from soil analyses. Putative fungal functional groups (e.g., arbuscular mycorrhiza fungi, plant pathogens and endophytes) were identified using FUNGuild (Nguyen et al. 2016).

\section{Statistical analyses}

To test whether soil-microbes-mediated apparent competition on alien invaders is more negative when the soil originates from multiple native species, we used mixed effect models to analyze the biomass production of the alien plants, as implemented in the nlme package (Pinheiro et al. 2018) with R 3.4.0 (R Core Team 2019). This analysis was restricted to the subset of 120 plants that grew in pots inoculated with soil samples from four different pots of the conditioning phase (Fig. 1a). The models included aboveground, belowground or total biomass as the response variables, and soil-diversity treatment (i.e., diversity-1, -2, and -4) as the fixed effect. The models included identity of the alien species and identity of the soil (i.e., identity of the native species for diversity-1, and identity of the species combination for diversity- 2 and -4 treatments) as random effects. Because initial data exploration showed that the effect of diversity is nonlinear, we included soil diversity as a categorical instead of as a continuous variable. To improve homoscedasticity of residuals, we allowed the alien species and soil diversity treatments to have different variances by using the varComb and varIdent functions (Zuur et al. 2009). We assessed the significance of soil-diversity treatment with likelihood-ratio tests by comparing models with and without the soil-diversity treatment (Zuur et al. 2009). We then performed multiple comparisons to test for pairwise differences among the three soil-diversity levels with the multicomp package (Hothorn et al. 2008). To reduce false discovery rate, we report adjusted $P$ values that were corrected with the single-step method in the multicomp package. In addition, because the effect of soil microbe can be species-specific
(Bever et al. 1997), we also tested the effect of soil-diversity on each alien species separately.

To test whether mixing soil samples affected growth of alien species, we used a similar analysis approach as for the test of soil-diversity effects. The only differences were that we used the subset of 120 alien plants that grew on soils from single species (i.e., diversity-1; Fig. 1b), and that we used the number of soil samples instead of soildiversity as the fixed effect. Forty pots of this data set were shared with the data set used to test soil-diversity effects (i.e., the pots of diversity-1 in Fig. 1a). Again, we tested the effect of number of soil samples on each alien species separately.

To test whether the five native plant species differently trained the soil microbial communities, we first used a linear model to analyze the alpha diversities of soil microbes. The models included species richness or Shannon indexes as the response variables, and plant species identity as the explanatory variables. We assessed the significance of differences among plant species with likelihood-ratio tests by comparing models with and without the factor "plant species identity." Second, we analyzed whether variation in composition of microbial communities was explained by plant species identity by using Bray-Curtis dissimilarities and permutational analysis of variance (PERMANOVA), as implemented in the adonis function of the vegan package (Oksanen et al. 2019). Before calculating the Bray-Curtis dissimilarities, we square-root transformed the reads so that rare ASVs had more weight (relative to their abundance) on the Bray-Curtis dissimilarities. We used nonmetric multidimensional scaling (NMDS) to illustrate differences in the composition of soil microbial communities of the plant species. Third, we determined shared and unshared taxa among plant species (based on ASVs occurring in at least one of the samples) and visualized them using 5sets-Venn diagrams with the eulerr package (Larsson 2018).

\section{RESUlTS}

\section{Microbial communities of trained soils}

We detected a total of 21,724 bacterial Amplicon Sequence Variants (ASVs) and 1,231 fungal ASVs. Identity of native plant species did not significantly explain Shannon diversity (Appendix S2: Table S1, Fig. S1). However, identity of native plant species marginally explained species richness of bacterial and fungal communities (Appendix S2: Table S1, Fig. S1), and largely explained the variation in composition of bacterial communities (Fig. 2a; PERMANOVA, $r^{2}=0.249, \quad F_{4,24}=1.990$, $P=0.001$ ) and that of fungal communities (Fig. 2b; PERMANOVA, $\left.r^{2}=0.195, F_{4,23}=1.391, P=0.001\right)$. In addition, $55.1 \%$ of bacterial ASVs and $61.4 \%$ of fungal ASVs were not shared among native plant species (Fig. 2d, e).

Fungal functional groups (i.e., AMF, plant pathogen and endophyte) could be assigned to 490 ASVs, 
representing $33.9 \%$ of the ITS sequence reads. Of these ASVs, 26 were identified as AMF, 101 as endophytes and 134 as plant pathogens (54 ASVs were identified as both plant pathogen and endophyte). Although identity of native plant species only marginally explained variation in composition of identified fungal endophytes (Appendix S2: Fig. S2; PERMANOVA, $r^{2}=0.193$, $F_{4,23}=1.370, P=0.083$; for AMF we had insufficient data), it largely explained variation in composition of identified fungal pathogens (Fig. 2c; PERMANOVA, $\left.r^{2}=0.164, F_{4,23}=1.307, P=0.034\right)$. In addition, $42.1 \%$ of fungal pathogen ASVs were not shared among native plant species (Fig. 2f).

\section{Biomass of plants in the test phase}

Diversity of the native species used to create the soil inocula affected the aboveground biomass production of the alien plants significantly $\left(\chi^{2}=7.956, P=0.019\right.$; Appendix S3: Table S1). The aboveground biomass of the alien plants was not significantly different between the diversity-1 and diversity-2 treatments (Fig. 3a; Appendix S3: Table S2; multiple comparison for diversity- 2 vs. diversity- $1, z=1.123, P=0.500$ ). In other words, it did not matter whether the soil inoculum came from one or two native species. However, the alien plants produced $11.7 \%$ less aboveground biomass in the diversity-4 treatment than in the diversity-2 treatment (Fig. 3a; diversity-4 vs. diversity-2, $\quad z=-2.964, \quad P=0.008$ ). Although the alien plants tended to also achieve less aboveground biomass $(-7.26 \%)$ in the diversity-4 treatment than in the diversity-1 treatment (Fig. 3a), the difference was not statistically significant (diversity-4 vs. diversity-1; $z=-1.638, P=0.230$ ). A similar pattern was found for total biomass, but there the effect of diversity-4 vs. diversity-2 was marginally significant (Appendix S3: Fig. S1, Table S2; diversity-4 vs. diversity$2, z=-2.067, P=0.096)$. Belowground biomass did not differ among the three diversity treatments (Appendix S3: Fig. S1, Table S1). In analyses of each alien plant species separately, we did not find significant effects of the diversity treatments on aboveground biomass (Appendix S4: Table S1). However, three out of the four species (Lolium multiflorum, Senecio inaequidens, and Vicia villosa) showed patterns similar to those of the analysis of all four species jointly (Appendix S4: Fig. S1). a) Bacteria

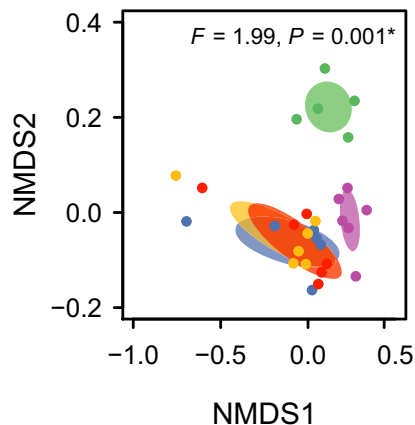

d) Bacteria

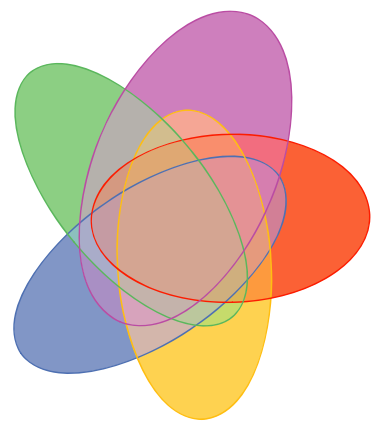

b) All fungi

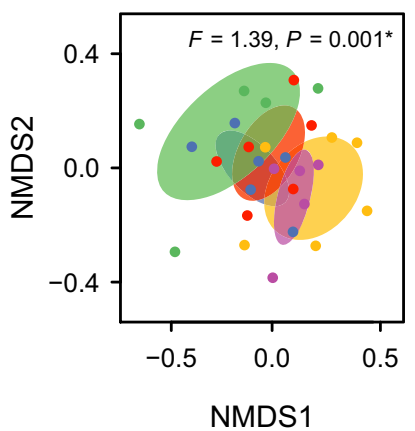

e) All fungi

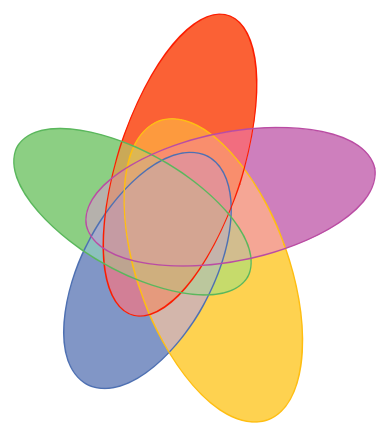

c) Fungal pathogens

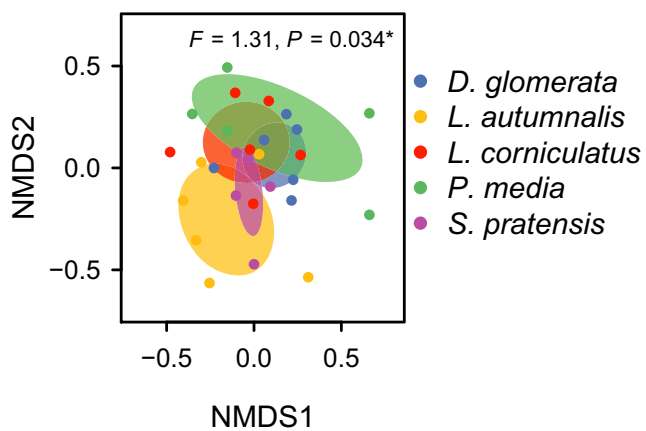

f) Fungal pathogens

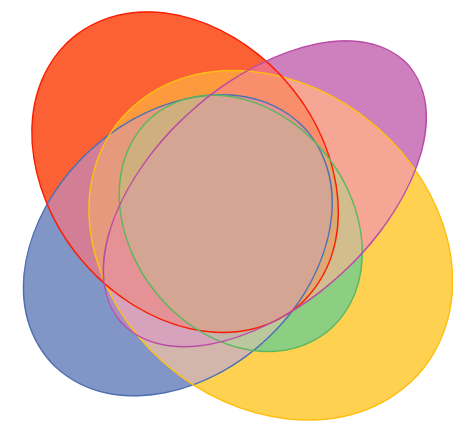

FIG. 2. Dissimilarity (Bray-Curtis dissimilarities and unshared amplicon sequence variants [ASVs]) of (a, d) bacterial, (b, e) fungal, and (c, f) fungal pathogen community composition among soils trained by different native species. Different colors represent different plant species. In the NMDS figures (upper panel), data points represent soil samples. Ellipses represent means \pm SDs for each native plant species. In the Venn diagrams (lower panel), the sizes of the circles and their overlapping area are proportional to the number of ASVs. [Color figure can be viewed at wileyonlinelibrary.com] 
We found that the approach of mixing soil samples per se did not affect the biomass production of the alien plants (Appendix S3: Table S3). When the alien plants grew on soils from a single native species, their aboveground biomass did not change with the number of pots from which the soil had been collected (Fig. 3b; Appendix S3: Table S4; four vs. two soil samples, $z=-0.296, \quad P=0.953$; four vs. one soil samples, $z=0.228, \quad P=0.972 ; \quad$ two vs. one soil samples, $z=0.519, P=0.862)$. Similar patterns were found for total and belowground biomass (Appendix S3: Fig. S1c, $\mathrm{d}$, Table S4), and for each alien plant species (Appendix S4: Table S2, Fig. S2).

\section{Discussion}

We here show that alien plants achieved less aboveground biomass when grown on a mixture of soil trained by four native species than when grown on a mixture of soil trained by two native species. So, whereas previous studies frequently ascribed the negative relationship between diversity and invasibility to resource competition (Byers and Noonburg 2003), we showed that this relationship could also be driven by soil-microbes-mediated apparent competition.

The negative effect of soil from species-rich native communities on alien plants could be mediated by the establishment of a diverse community of soil pathogens during the conditioning phase. This is indicated by our finding that composition of fungal pathogen communities in their rhizospheres differed among native plant species. Interestingly, the difference in composition of fungal pathogen communities among plant species was mainly driven by rare pathogens, as the difference was more apparent when we put more weight on the rare pathogens (i.e., by square-root transforming reads of

a

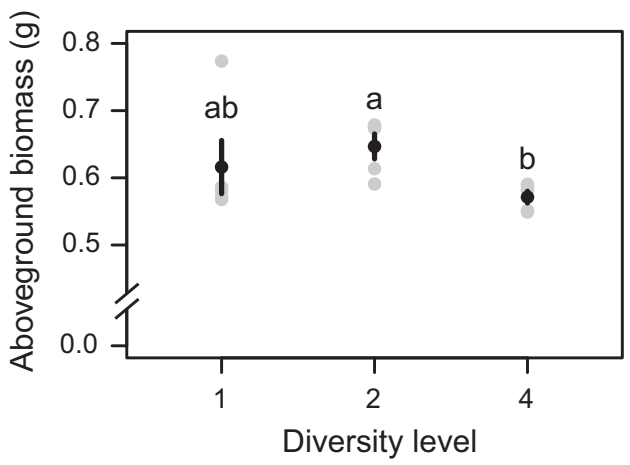

pathogens when calculating the Bray-Curtis dissimilarity; see Appendix S2: Fig. S4 for the results that are based on untransformed data). Taken together, plant communities that contained multiple native species could harbor a greater diversity of plant pathogens, some of which might be rare but have strong negative impact on the alien plant.

The preceding conclusion thus supports the amplification-effect hypothesis (Keesing et al. 2006), and does not support the predictions of the dilution-effect hypothesis (Schmidt and Ostfeld 2001, Ostfeld and Keesing 2012) and the enemy-release hypothesis (Mitchell and Power 2003). One explanation for the latter could be that, although alien plants could escape from their co-evolved enemies (i.e., specialist enemies), they might still encounter biotic resistance from generalist enemies (i.e., spillover; Maron and Vilà 2001, Dawson et al. 2014, Zhang et al. 2018). This explanation becomes more plausible given that the dilution effect is less likely to happen when pathogens are generalists (Power and Mitchell 2004). Therefore, our finding that species-rich communities are more resistant to biological invasion might be driven by generalist pathogens. It needs to be kept in mind that mutualists (e.g., arbuscular mycorrhizal fungi) are also important in determining soil-microbes-mediated effects and invasion success (Bever et al. 1997, Richardson et al. $2000 a$ ). Detailed investigation of mutualists, such as targeting arbuscular-mycorrhizal-fungi using specific primers, could provide new insights.

Although species-rich communities could suppress alien plant performance through modifying soil microbial communities, this effect was only significant when we compared the alien plants grown on soils trained by four native species with those grown on soils trained by two native species. The comparisons with plants grown on soils trained by only one native species (i.e., diversity-1 vs.

\section{b}

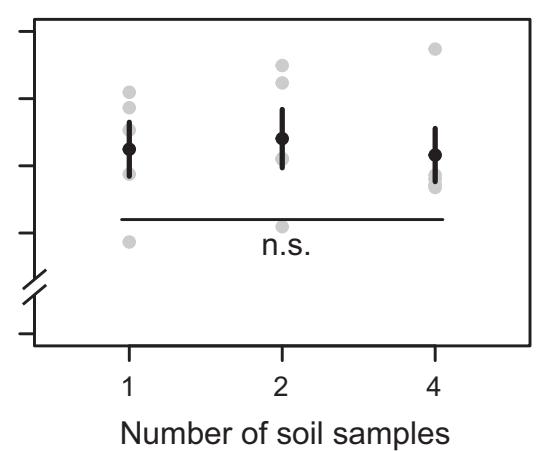

FIG. 3. Aboveground biomass (means $\pm \mathrm{SEs}$ ) of alien plants (a) when their pots had been inoculated with soil trained by one, two, or four native species and (b) when their pots had been inoculated with soil samples trained by one, two, or four individuals of the same species. Gray dots represent mean values of aboveground biomass of alien plants when grown on soil trained by different native species or by different native species combinations. Different letters above the error bars indicate significant differences $(P<0.05)$ between different treatments based on Tukey's multiple comparison; n.s. indicates no significant differences among treatments. 
diversity-2, and diversity-1 vs. diversity-4) were not significant. Probably, this is because at low diversity, the variation among native species in their effects on the alien species was large (see error bars of diversity-1 in Fig. 3). As we have a relative low number of replicates (i.e., five native species), the difference between diversity-1 and other diversity levels might become more apparent when using more native species. In addition, invasion is a probabilistic process (Crawley 1987, Levine and D'Antonio 1999), and the probability that a community includes native species that can impede alien plants increases with the diversity of the community. Such a selection effect (Huston 1997) is also indicated by the $14.9 \%$ higher variance in the diversity- 1 treatment than diversity-2 and -4 treatments. If most native species do not strongly affect the alien plants, it will, however, be difficult to detect a diversity effect at low diversities. This could explain the absence of a difference in biomass production of aliens between the diversity- 1 and diversity- 2 treatments.

Unexpectedly, root biomass of alien plants did not change with diversity of native communities. Possibly, our finding may reflect that the alien plants have high phenotypic plasticity (Davidson et al. 2011), and changed their root-shoot allocation in such a way that they could maintain maximum root biomass under different conditions. However, we did not find a significant response of root weight ratio to diversity of native communities (Appendix S3: Table S2). An alternative explanation could be that roots in all diversity treatments were limited by pot size. Poorter et al. (2012) found that pot limitation starts to happen when total plant biomass per unit pot volume exceeded $1 \mathrm{~g} / \mathrm{L}$. In our study, the total plant biomass per unit pot volume was $1.45 \mathrm{~g} / \mathrm{L}$ on average. Although this value is lower than in most pot experiments analyzed by Poorter et al. (2012), it indicates that roots were slightly bounded.

When growing alien plants on soil from a single native species, we found that aboveground biomass of the alien plants did not change with the number of individuals (i.e., pots) from which the trained soil came. The absence of such an effect could reflect that in our study, genetic variation among the individuals of a native species was likely to be low, because the seeds used for each species came from the same origin. In addition, the field where we collected the soil used as inoculum of live soil biota was relatively homogeneous. Possibly, if genetic variation of the species used for soil conditioning would be high or if the composition of soil biota varies substantially across small spatial scales, it might matter whether one mixes soil samples from different individuals or not. Therefore, we recommend always accounting for potential mixing effects in diversity studies by using soil samples from an equal number of plant individuals.

\section{Future directions}

Invasibility refers to the probability of invaders to establish in native communities. Therefore, it is determined by two stages: (1) the probability of germination of the invaders, and (2) if germinated, the probability of maintaining persistent populations. We tested individual growth, which is likely to positively correlate with population growth and thus the probability of maintaining persistent populations. However, as the magnitude and direction of soil-mediated apparent competition can vary across different life stages (Dudenhöffer et al. 2018), more comprehensive tests require also longer-term investigations that consider all life stages (e.g., germination and reproduction).

We found that the diversity- 4 treatment reduced biomass of alien species by $11.7 \%$ (95\% CI: $3.7-20.1 \%)$. It remains unknown whether this reduction is sufficient to eliminate the dominance of alien species over natives. Previous multispecies experiments offer us some clues showing that native species produced $13.6-38.3 \%$ less biomass than aliens (Godoy et al. 2011, Zhang and van Kleunen 2019). Therefore, the dominance of alien species over natives would be alleviated if native species are less limited by the negative effects from native communities than aliens are. As we know little about whether native and alien species respond differently to soil-microbes-mediated apparent competition, direct tests that include both alien and native species in the test phase are needed.

The soil that we used to create the different diversity levels came from pots in which plants were grown without direct interaction (e.g., resource competition). It could be argued that the lack of direct plant-plant interactions in the soil-conditioning phase could have biased our results (Leff et al. 2018). For example, resource competition can affect plant growth and possibly root exudation, which may change the microbial community, and thus the effects on alien plants in the test phase. By mixing soil trained by different species rather than directly using soil trained by communities that consist of multiple species, we removed the effect of resource competition on the microbial community. Nevertheless, our method allowed us to isolate the effect of diversity that resulted from soil-microbes-mediated apparent competition. Still, research that includes direct interaction is needed to identify whether and how resource competition modifies diversity effect mediated by soil microbes.

\section{Conclusions}

Sixty years after Elton (1958) proposed the diversityinvasibility hypothesis, mounting evidence has shown that, at the local scale, species-rich communities are better at resisting invasion by alien plants (Beaury et al. 2020; but see Peng et al. 2019). However, few studies have tested the mechanisms underlying the diversity-invasibility relationship. Here, we revealed soil-microbemediated apparent competition as one mechanism. As invasibility is an emergent property of multiple types of interactions, a next step would be to test the relative importance of soil-microbes-mediated apparent 
competition and other processes, such as resource competition, in determining the diversity-invasibility relationship.

\section{ACKNOWLEDGMENTS}

We thank O. Ficht, M. Fuchs, S. Gommel, E. Mamonova, V. Pasqualetto, C. Rabung, B. Rüter, H. Vahlenkamp, and E. Werner for practical assistance and X. Liu for comments on a previous version. Z. Zhang acknowledges funding from the China Scholarship Council (201606100049) and support from the International Max Planck Research School for Organismal Biology. Y. Liu acknowledges funding from the Chinese Academy of Sciences (Y9H1011001, Y9B7041001). The authors declare no conflict of interest. Z. Zhang, Y. Liu, and M. van Kleunen designed the experiment. Z. Zhang and Y. Liu performed the experiment. C. Brunel led the soil analyses. $Z$. Zhang analyzed the data and led the writing with input from all others.

\section{Literature Cited}

Beaury, E. M., J. T. Finn, J. D. Corbin, V. Barr, and B. A. Bradley. 2020. Biotic resistance to invasion is ubiquitous across ecosystems of the United States. Ecology Letters 23:476-482.

Bennett, J. A., and J. Klironomos. 2019. Mechanisms of plantsoil feedback: interactions among biotic and abiotic drivers. New Phytologist 222:91-96.

Bever, J. D., K. M. Westover, and J. Antonovics. 1997. Incorporating the soil community into plant population dynamics: the utility of the feedback approach. Journal of Ecology 85:561-573.

Brunel, C., Y. Beifen, R. Pouteau, J. Li, and M. van Kleunen. 2019. Responses of rhizospheric microbial communities of native and alien plant species to Cuscuta parasitism. Microbial Ecology 79:617-630.

Bundesamt für Naturschutz. 2003. FloraWeb: Daten und Informationen zu Wildpflanzen und zur Vegetation Deutschlands. http://www.floraweb.de/

Byers, J. E., and E. G. Noonburg. 2003. Scale dependent effects of biotic resistance to biological invasion. Ecology 84:1428-1433.

Callahan, B. J., P. J. McMurdie, and S. P. Holmes. 2017. Exact sequence variants should replace operational taxonomic units in marker-gene data analysis. ISME Journal 11:2639-2643.

Callahan, B. J., P. J. McMurdie, M. J. Rosen, A. W. Han, A. J. A. Johnson, and S. P. Holmes. 2016. DADA2: High-resolution sample inference from Illumina amplicon data. Nature Methods 13:581-583.

Case, T. J. 1990. Invasion resistance arises in strongly interacting species-rich model competition communities. Proceedings of the National Academy of Sciences USA 87:9610-9614.

Crawley, M. J. 1987. What makes a community invasible? Pages 429-451 in M. J. Crawley and P. J. Edwards, editors. Colonization, succession and stability. Blackwell Scientific, Oxford, UK.

Davidson, A. M., M. Jennions, and A. B. Nicotra. 2011. Do invasive species show higher phenotypic plasticity than native species and if so, is it adaptive? A meta-analysis. Ecology Letters 14:419-431.

Dawson, W., et al. 2017. Global hotspots and correlates of alien species richness across taxonomic groups. Nature Ecology \& Evolution 1:0186.

Dawson, W., A. Bottini, M. Fischer, M. van Kleunen, and E. Knop. 2014. Little evidence for release from herbivores as a driver of plant invasiveness from a multi-species herbivore-removal experiment. Oikos 123:1509-1518.
Decaëns, T. 2010. Macroecological patterns in soil communities. Global Ecology and Biogeography 19:287-302.

Dudenhöffer, J.-H., A. Ebeling, A.-M. Klein, and C. Wagg. 2018. Beyond biomass: soil feedbacks are transient over plant life stages and alter fitness. Journal of Ecology 106:230-241.

Elton, C. S. 1958. The ecology of invasion by animals and plants. The University of Chicago Press, Chicago, Illinois, USA.

Godoy, O., F. Valladares, and P. Castro-Díez. 2011. Multispecies comparison reveals that invasive and native plants differ in their traits but not in their plasticity. Functional Ecology 25:1248-1259.

Gundale, M. J., D. A. Wardle, P. Kardol, and M. C. Nilsson. 2019. Comparison of plant-soil feedback experimental approaches for testing soil biotic interactions among ecosystems. New Phytologist 221:577-587.

Heinen, R., M. van der Sluijs, A. Biere, J. A. Harvey, and T. M. Bezemer. 2018. Plant community composition but not plant traits determine the outcome of soil legacy effects on plants and insects. Journal of Ecology 106:1217-1229.

Holt, R. D. 1977. Predation, apparent competition, and the structure of prey communities. Theoretical Population Biology 12:197-229.

Hothorn, T., F. Bretz, and P. Westfall. 2008. Simultaneous inference in general parametric models. Biometrical Journal 50:346-363.

Hudson, P. J., A. P. Dobson, and K. D. Lafferty. 2006. Is a healthy ecosystem one that is rich in parasites? Trends in Ecology \& Evolution 21:381-385.

Hurlbert, S. H. 1984. Pseudoreplication and the design of ecological field experiments. Ecological Monographs 54:187211.

Huston, M. A. 1997. Hidden treatments in ecological experiments: re-evaluating the ecosystem function of biodiversity. Oecologia 110:449-460.

in 't Zandt, D., A. van den Brink, H. de Kroon, and E. J. W. Visser. 2019. Plant-soil feedback is shut down when nutrients come to town. Plant and Soil 439:541-551.

Keesing, F., R. D. Holt, and R. S. Ostfeld. 2006. Effects of species diversity on disease risk. Ecology Letters 9:485-498.

Klindworth, A., E. Pruesse, T. Schweer, J. Peplies, C. Quast, M. Horn, and F. O. Glockner. 2013. Evaluation of general 16S ribosomal RNA gene PCR primers for classical and next-generation sequencing-based diversity studies. Nucleic Acids Research 41:e1.

Kulmatiski, A., K. H. Beard, J. R. Stevens, and S. M. Cobbold. 2008. Plant-soil feedbacks: a meta-analytical review. Ecology Letters 11:980-992.

Larsson, J.2018. eulerr: area-proportional Euler and Venn diagrams with ellipses. 3 .

Leff, J. W., et al. 2018. Predicting the structure of soil communities from plant community taxonomy, phylogeny, and traits. ISME Journal 12:1794-1805.

Levine, J. M. 2000. Species diversity and biological invasions: relating local process to community pattern. Science $288: 852$ 854.

Levine, J. M., P. B. Adler, and S. G. Yelenik. 2004. A meta-analysis of biotic resistance to exotic plant invasions. Ecology Letters 7:975-989.

Levine, J. M., and C. M. D’Antonio. 1999. Elton revisited: a review of evidence linking diversity and invasibility. Oikos 87:15-26.

Maron, J. L., and M. Vilà. 2001. When do herbivores affect plant invasion? Evidence for the natural enemies and biotic resistance hypotheses. Oikos 95:361-373.

Martin, M. 2011. Cutadapt removes adapter sequences from high-throughput sequencing reads. EMBnet.journal 17: $10-13$. 
Mitchell, C. E., et al. 2006. Biotic interactions and plant invasions. Ecology Letters 9:726-740.

Mitchell, C. E., and A. G. Power. 2003. Release of invasive plants from fungal and viral pathogens. Nature 421:625-627.

Morris, W. F., et al. 2007. Direct and interactive effects of enemies and mutualists on plant performance: a meta-analysis. Ecology 88:1021-1029.

Müller, G., M. van Kleunen, and W. Dawson. 2016. Commonness and rarity of alien and native plant species - the relative roles of intraspecific competition and plant-soil feedback Oikos 125:1458-1466.

Nguyen, N. H., Z. Song, S. T. Bates, S. Branco, L. Tedersoo, J. Menke, J. S. Schilling, and P. G. Kennedy. 2016. FUNGuild: an open annotation tool for parsing fungal community datasets by ecological guild. Fungal Ecology 20:241-248.

Nilsson, R. H., et al. 2018. The UNITE database for molecular identification of fungi: handling dark taxa and parallel taxonomic classifications. Nucleic Acids Research 47:D259-D264.

Oksanen, J., et al. 2019. The vegan package. https://cran.r-pro ject.org, https://github.com/vegandevs/vegan

Orgiazzi, A., E. Lumini, R. H. Nilsson, M. Girlanda, A. Vizzini, P. Bonfante, and V. Bianciotto. 2012. Unravelling soil fungal communities from different Mediterranean land-use backgrounds. PLoS ONE 7:e34847.

Ostfeld, R. S., and F. Keesing. 2012. Effects of host diversity on infectious disease. Annual Review of Ecology, Evolution, and Systematics 43:157-182.

Peacher, M. D., and S. J. Meiners. 2020. Inoculum handling alters the strength and direction of plant-microbe interactions. Ecology 101:e02994.

Peng, S., N. L. Kinlock, J. Gurevitch, and S. Peng. 2019. Correlation of native and exotic species richness: a global metaanalysis finds no invasion paradox across scales. Ecology 100 e02552.

Pinheiro, J., D. Bates, S. DebRoy, D. Sarkar, and R Core Team. 2018. Linear and nonlinear mixed effects models. $\mathrm{R}$ package version 3:57. https://CRAN.R-project.org/package $=$ nlme

Poorter, H., J. Bühler, D. van Dusschoten, J. Climent, and J. A. Postma. 2012. Pot size matters: a meta-analysis of the effects of rooting volume on plant growth. Functional Plant Biology 39:839-850.

Power, A. G., and C. E. Mitchell. 2004. Pathogen spillover in disease epidemics. American Naturalist 164(Suppl 5):S79-S89.

Pyšek, P., et al. 2019. Facultative mycorrhizal associations promote plant naturalization worldwide. Ecosphere 10:e2937.
Pyšek, P., J. Pergl, F. Essl, B. Lenzner, W. Dawson, H. Kreft, P. Weigelt, M. Winter, J. Kartesz, and M. Nishino. 2017. Naturalized alien flora of the world. Preslia 89:203-274.

Quast, C., E. Pruesse, P. Yilmaz, J. Gerken, T. Schweer, P. Yarza, J. Peplies, and F. O. Glöckner. 2013. The SILVA ribosomal RNA gene database project: improved data processing and web-based tools. Nucleic Acids Research 41: D590-D596.

R Core Team. 2019. R: a language and environment for statistical computing. R Foundation for Statistical Computing, Vienna, Austria. http://www.R-project.org/

Reinhart, K. O., and R. M. Callaway. 2006. Soil biota and invasive plants. New Phytologist 170:445-457.

Reinhart, K. O., and M. J. Rinella. 2016. A common soil handling technique can generate incorrect estimates of soil biota effects on plants. New Phytologist 210:786-789.

Richardson, D. M., N. Allsopp, C. M. D'Antonio, S. J. Milton, and M. RejmÁNek. 2000a. Plant invasions-the role of mutualisms. Biological Reviews 75:65-93.

Richardson, D. M., P. Pyšek, M. Rejmánek, M. G. Barbour, F. D. Panetta, and C. J. West. 2000b. Naturalization and invasion of alien plants: concepts and definitions. Diversity and Distributions 6:93-107.

Rinella, M. J., and K. O. Reinhart. 2018. Toward more robust plant-soil feedback research. Ecology 99:550-556.

Schmidt, K. A., and R. S. Ostfeld. 2001. Biodiversity and the dilution effect in disease ecology. Ecology 82:609-619.

Seebens, H., et al. 2018. Global rise in emerging alien species results from increased accessibility of new source pools. Proceedings of the National Academy of Sciences USA 115: E2264-E2273.

van Kleunen, M., W. Dawson, O. Bossdorf, and M. Fischer. 2014. The more the merrier: multi-species experiments in ecology. Basic and Applied Ecology 15:1-9.

Zhang, Z., X. Pan, D. Blumenthal, M. van Kleunen, M. Liu, and B. Li. 2018. Contrasting effects of specialist and generalist herbivores on resistance evolution in invasive plants. Ecology 99:866-875.

Zhang, Z., and M. van Kleunen. 2019. Common alien plants are more competitive than rare natives but not than common natives. Ecology Letters 22:1378-1386.

Zuur, A., E. Ieno, N. Walker, A. Saveliev, and G. Smith. 2009. Mixed effects models and extensions in ecology with R. Spring Science and Business Media, New York, New York, USA.

\section{SUPPORTING INFORMATION}

Additional supporting information may be found in the online version of this article at http://onlinelibrary.wiley.com/doi/ 10.1002/ecy.3187/suppinfo

\section{Data Availability}

Data associated with this study are available from the Dryad Digital Repository: https://doi.org/10.5061/dryad.wm37pvmk6 